\section{The distinction between mental and physical illness}

\author{
R. E. KENDELL
}

Conditions that now would be regarded as 'mental illnesses', such as mania, melancholia and hysteria, have figured in classifications of disease since the time of Hippocrates, and for over 2000 years were treated by physicians with much the same range of potions, medicaments and attempts to correct humoral imbalance as they employed for other more obviously medical disorders. Although Plato attributed some forms of madness to the Gods, and medieval theologians like Thomas Aquinas attributed hallucinations and insanity to demons and other supernatural influences, from the Renaissance to the second half of the 18th century melancholia and other forms of insanity were generally regarded as bodily illnesses, not differing in any fundamental way from other diseases. When the mid18th century belle lettriste Lady Mary Wortley Montagu commented that "madness is as much a corporeal distemper as the gout or asthma", she was simply expressing the "commonplace of high and low, lay and medical opinion alike" (Porter, 1987).

\section{ORIGINS OF \\ THE DISTINCTION}

The idea that insanity was fundamentally different from other illnesses, that it was a disease of the mind rather than the body, only developed towards the end of the 18th century. The scene was set by Cartesian dualism, the dominant philosophical influence of the time, but medical opinion and medical impotence also played crucial roles. The development first of private mad-houses and later of large, purpose-built lunatic asylums took the management of the insane out of the hands of the general run of physicians; and because the managers of these new institutions were concerned only with insanity it was relatively easy for them to regard it as different from other illnesses that did not concern them. At the same time it was becoming clear that insanity was not accompanied by the obvious pathological changes that post-mortem examination was revealing in other diseases. It was increasingly apparent also that although the armamentarium of 18th century medicine - special diets, bleeding, purging, emetics and blistering - was as effective in the management of hypochondriasis and hysteria as it was in other disorders, it had little effect on madness itself. In England the success of the clergyman Francis Willis in curing the King (George III) of his madness after the conspicuous failure of his physicians to do so, and the remarkable success of the York Retreat (opened by the Quaker William Tuke in 1796) in calming and curing its inmates despite using few medicaments or restraints, both had a considerable influence on public opinion. It was in this climate that the terms "disease of the mind", "disorder of the mind" and "mental illness" first began to be widely used. Indeed, the York Retreat was explicitly for "persons afflicted with disorders of the mind" (Hunter \& Macalpine, 1963).

The implication of these new terms was that madness was a disease of the mind, not of the body, and there was some debate whether diseased minds might not be better treated by philosophers than by physicians. 'Moral treatment' - a benevolent, ordered regime based on moderation and religious observance rather than medication - became the mainstay of the new asylums that were built in the early years of the 19th century, and initially several of them had no physician. It was not long, though, before the medical profession reasserted itself. In Philadelphia, Benjamin Rush (1812) insisted that the fundamental pathology of what he himself referred to as "disease of the mind" was somatic (he suggested that it lay "primarily in the blood vessels of the brain") and in 1845 Wilhelm Griesinger, the first professor of psychiatry, convinced most of his German contemporaries when he argued in his influential textbook that "Psychische Krankheiten sind Erkrankungen des Gehirns" (mental illnesses are diseases of the brain). By the middle of the 19th century it was also generally accepted that the superintendent of any properly run lunatic asylum should be a physician. But the terms mental illness and mental disease survived, partly because they clearly implied that what had previously been called madness or insanity was medical territory. The doubts about causation also survived, even within the medical profession itself. Indeed, the school of psychoanalysis that emerged at the end of the 19th century regarded all mental illnesses as entirely psychogenic disorders to be treated by psychotherapy; and until the 1930s mental disease remained largely uninfluenced by the physician's pharmacopoeia and, apart from general paresis, could not be shown to be accompanied by any cerebral pathology, macroscopic or microscopic.

The failure to find any somatic pathology underlying many of the disorders that they treated affected neurologists as well as psychiatrists. Reynolds (1855) responded to this problem by distinguishing between 'organic' and 'functional' disorders. The former possessed a somatic pathology and the latter did not, and Reynolds assumed that the symptoms of functional disorders were due either to a decrease or an inappropriate increase in functional activity. Subsequently, the terms organic and functional came to be used by his successors simply to distinguish between conditions in which somatic pathology had and had not been demonstrated. Gowers' textbook, for example, classified Parkinson's disease, chorea, torticollis, epilepsy and narcolepsy as functional simply because at the time there was no visible lesion to justify regarding them as organic (Gowers, 1893). The terms were then adopted by psychiatrists, mainly as a convenient way of distinguishing dementias and confusional states in which a somatic pathology was apparent from affective, schizophrenic and paranoid psychoses in which no such pathology could be demonstrated. Unfortunately, the ambiguity inherent in the term functional was also taken over, with the result that some psychiatrists assumed that it implied simply that no somatic pathology had yet been demonstrated whereas others assumed that the disorders so labelled were purely mental and that they had no somatic 
pathology. (Contemporary neurologists still use the term functional, usually informally and nearly always to imply their conviction that the patient's symptoms are 'psychogenic'.)

\section{MENTAL AND PHYSICAL ILLNESS IN CONTEMPORARY MEDICINE}

A distinction between mental and physical illness is still made, both by the lay public and by many doctors, and the terms 'mental disorder' and 'mental and behavioural disorder' are still used in the two most widely used official nomenclatures, the World Health Organization's International Classification of Diseases (ICD) and the American Psychiatric Association's Diagnostic and Statistical Manual (DSM). This has the unfortunate effect of helping to perpetuate two assumptions that have long since been abandoned by all thinking physicians, namely that mental disorders are disorders of the mind rather than the body, and that they are fundamentally different from other illnesses.

In reality, neither minds nor bodies develop illnesses. Only people (or, in a wider context, organisms) do so, and when they do both mind and body, psyche and soma, are usually involved. Pain, the most characteristic feature of so-called bodily illness, is a purely psychological phenomenon, and the first manifestation of most infections, from influenza to plague, is also a subjective change - a vague general malaise (Canter, 1972). Fear and other emotions play an important role in the genesis of myocardial infarction, hypertension, asthma and other bodily illnesses, and bodily changes such as fatigue, anorexia and weight loss are commonplace in psychiatric disorders. That most characteristic of all psychiatric disorders, depressive illness, illustrates the impossibility of distinguishing between physical and mental illnesses.

There is good evidence from both family and twin studies (Andreasen et al, 1986; Kendler et al, 1992) that genetic factors make an important contribution to the aetiology of the whole range of depressive disorders, from the mildest to the most severe. This necessarily implies that there must be innate biological differences between those who are and are not prone to depression, and this is confirmed by the fact that drugs that have no effect on mood in normal people relieve depression in those who are ill, and the observation that a depressed mood can be precipitated in people who are prone to depressive illnesses simply by manipulating the tryptophan content of their diet (Delgado et al, 1990). There is unassailable evidence, therefore, of somatic abnormalities in this most typical and common of mental illnesses. Analogous evidence could be presented for schizophrenia, obsessional disorder and panic disorder. Indeed, in the case of schizophrenia there is extensive evidence of widespread, albeit subtle, brain pathology as well as strong evidence of genetic transmission.

The fact is, it is not possible to identify any characteristic features of either the symptomatology or the aetiology of so-called mental illnesses that consistently distinguish them from physical illnesses. Nor do socalled physical illnesses have any characteristics that distinguish them reliably from mental illnesses. If pathological changes and dysfunctions are restricted to organs other than the brain, as is often the case, effects on mentation and behaviour are relatively restricted, but this is an inconstant and purely quantitative difference, and in any case does not apply to diseases of the brain or situations in which there is a secondary disturbance of cerebral function. There are many differences between 'mental' and 'physical' disorders, of course. Hallucinations, delusions and grossly irrational behaviour, for example, are a conspicuous feature of the former. But they occur only in a small proportion of mental disorders, and also feature in the confusional states that may complicate many physical disorders. The mechanisms underlying hysterical amnesia or paraplegia are very different from those underlying the amnesia of dementia or the paraplegia of spinal injury and are commonly described as 'psychogenic'. But a myocardial infarction precipitated by fear or anger is equally 'psychogenic', and in both cases there are good grounds for assuming that the emotional predicament generates neuronal or endocrine changes that play a critical role in producing the loss of access to memories, loss of voluntary movement or inadequate oxygenation of the myocardium. In reality, the differences between mental and physical illnesses, striking though some of them are, are quantitative rather than qualitative, differences of emphasis rather than fundamental differences, and no more profound than the differences between diseases of the circulatory system and those of the digestive system, or between kidney diseases and skin diseases.

Why then do we still talk of 'mental' illnesses, or indeed of 'physical' illnesses? The answer is provided in the introduction to the current (1994) edition of the Diagnostic and Statistical Manual of Mental Disorders (DSM-IV): "The term mental disorder unfortunately implies a distinction between 'mental' disorders and 'physical' disorders that is a reductionistic anachronism of mind/body dualism. A compelling literature documents that there is much 'physical' in 'mental' disorders and much 'mental' in 'physical' disorders. The problem raised by the term 'mental disorders' has been much clearer than its solution, and, unfortunately, the term persists in the title of DSM-IV because we have not found an appropriate substitute".

\section{MENTAL AND PHYSICAL DISORDERS IN CONTEMPORARY CLASSIFICATIONS}

Against this background it is instructive to examine the status of mental and physical disorders, and the allocation of individual syndromes to broad groupings of disease, in contemporary classifications of disease.

The International Classification of Diseases is by far the most widely used comprehensive classification. It is important to appreciate, though, that it is not a textbook of medicine, sanctified by international approval. Its status and role are more modest; it is essentially "a statistical classification of diseases and other health problems, to serve a wide variety of needs for mortality and health-care data" (World Health Organization, 1992). Like its predecessors, the current revision, ICD-10, does not draw a fundamental distinction between mental and physical diseases. "Mental and behavioural disorders" (F00-99) are simply the fifth of seventeen categories of disease (World Health Organization, 1992). Several of these seventeen broad groupings (e.g. infectious and parasitic diseases; neoplasms; and congenital malformations, deformations and chromosomal abnormalities) are based on aetiology. Others (e.g. diseases of the circulatory system; diseases of the respiratory system) are based on the organ system primarily affected. Some (e.g. conditions 
originating in pregnancy, childbirth and the puerperium; and possibly mental and behavioural disorders as well) are heterogeneous and determined mainly by the medical speciality primarily responsible for treatment. None of the three underlying principles consistently takes precedence over the other two. Carcinoma of the bronchus (C34), for example, is classified by its aetiology - as a neoplasm - rather than as a disease of the respiratory system. Vascular dementia (F01), on the other hand, is classified as a mental disorder, despite the fact that in aetiological terms it is explicitly a vascular disorder. The distinction between diseases of the nervous system (G00-99) and mental and behavioural disorders (F00-99) is particularly illuminating. Most diseases of the brain, such as encephalitis and epilepsy, are classified as diseases of the nervous system. Others, like the postencephalitic and postconcussional syndromes, are classified as mental disorders. Some, like Alzheimer's and Parkinson's diseases, are listed as diseases of the nervous system and also, if they lead to dementia (as Alzheimer's disease, of course, invariably does), as mental diseases.

The American Psychiatric Association's Diagnostic and Statistical Manual, like other classifications produced by professional bodies, is essentially a classification of the disorders seen and treated by contemporary American psychiatrists and clinical psychologists. If, for example, child psychiatrists are asked to treat defiant, disobedient adolescents, as they are, the Manual has to contain a category-oppositional defiant disorder - for such patients. The same reasoning applies to substance-related disorders, somatoform disorders and sleep disorders, and would do so even without the statement quoted above, making it clear that the Association regards the distinction between mental and physical disorders as a meaningless anachronism.

Overall, it seems clear that in both ICD10 and DSM-IV, the two most widely used classifications of so-called mental disorders, the allocation of individual disorders to broad categories of disease or disorder is determined to a considerable extent by practical considerations - mainly which kind of medical specialist usually treats patients presenting with the syndrome in question - rather than by fundamental aetiological considerations. This is particularly true of the distinction between mental diseases and diseases of the (central) nervous system in ICD-10, which reflects little more

R. E. KENDELL, FRCPsych, Department of Psychiatry, Edinburgh University, 3 West Castle Road, Edinburgh EHIO 5AT, UK

(First received I August 2000, final revision 2 January 200I, accepted 22 January 200I)

than a pragmatic distinction between conditions generally treated by psychiatrists and cerebral disorders usually treated by neurologists.

\section{PUBLIC ATTITUDES}

Unfortunately, the linguistic distinction between mental and physical illnesses, and the mind/body distinction from which this was originally derived, still encourages many lay people, and some doctors and other health professionals, to assume that the two are fundamentally different. Both are apt to assume that developing a 'mental illness' is evidence of a certain lack of moral fibre and that, if they really tried, people with illnesses of this kind ought to be able to control their anxieties, their despondency and their strange preoccupations and 'snap out of it'. It is true, of course, that most of us believe in 'free will'; we believe that we ourselves and other people can exercise a certain amount of control over our feelings and behaviour. But there is no reason, justified either by logic or by medical understanding, why people suffering from, say, phobic anxiety or depression should be able to exert more control over their symptoms than those suffering from myxoedema or migraine. There is a further and equally damaging assumption that the symptoms of mental disorders are in some sense less 'real' than those of physical disorders with a tangible local pathology. As a result, people experiencing intense fatigue, or pain that is not accompanied by any obvious local lesion, are often dismayed or affronted by being told that they are suffering from neurasthenia, the chronic fatigue syndrome or 'psychogenic' pain, and interpret such diagnoses as implying that their doctor does not believe that they are really in pain or exhausted by the slightest effort, and is dismissing their complaints as 'all in the mind'.

\section{CONCLUSIONS}

Misunderstandings of this kind are important and frequent. They undermine the relationship between doctor and patient and often result in a refusal to consult a psychiatrist or clinical psychologist, or to countenance a potentially effective treatment. The answer to such problems lies in painstaking explanation and gentle persuasion, and in the longer run in better education of both the general public and doctors themselves, not in conniving with patients' convictions that their symptoms are caused by 'real' or 'physical' illnesses. It may be sensible sometimes to do this as a holding tactic in an individual patient. It is never appropriate in other contexts. Not only is the distinction between mental and physical illness ill-founded and incompatible with contemporary understanding of disease, it is also damaging to the long-term interests of patients themselves. It invites both them and their doctors to ignore what may be important causal factors and potentially effective therapies; and by implying that illnesses so described are fundamentally different from all other types of ill-health, it helps to perpetuate the stigma associated with 'mental' illness. We should talk of psychiatric illnesses or disorders rather than of mental illnesses; and if we do continue to refer to 'mental' and 'physical' illnesses we should preface both with 'so-called', to remind ourselves and our audience that these are archaic and deeply misleading terms.

\section{REFERENCES}

American Psychiatric Association (1994) Diagnostic and Statistical Manual of Mental Disorders (4th edn) (DSM-IV).Washington, DC: APA

Andreasen, N. C., Scheftner, W., Reich, T., et al (1986)

The validation of the concept of endogenous depression - a family study approach. Archives of General Psychiatry, 43, 246-25I.

Canter, A. (1972) Changes in mood during incubation of acute febrile disease and the effects of pre-exposure psychologic status. Psychosomatic Medicine, 34 424-430.

Delgado, P. L., Charney, D. S., Price, L. H., et al (1990) Serotonin function and the mechanism of antidepressant action: reversal of antidepressantinduced remission by rapid depletion of plasma tryptophan. Archives of General Psychiatry, 47, 4II-4I8.

Gowers, W. R. (1893) A Manual of Diseases of the Nervous System, Vol. I. Reprinted 1970. New York: Hafner. 
Griesinger, W. (1845) Pathologie und Therapie der Psychischen Krankheiten. Reprinted (1867) as Mental Pathology and Therapeutics (trans. C. L. Robertson \& J. Rutherford). London: The New Sydenham Society

Hunter, R. \& Macalpine, I. (1963) Three Hundred Years

of Psychiatry 1535-1860. London: Oxford University Press.

Kendler, K. S., Neale, M. C., Kessler, R. C., et al (1992) A population-based twin study of major depression in women: the impact of varying definitions of illness. Archives of General Psychiatry, 49, 257-266.

Porter, R. (1987) Mind-forg'd Manacles, p. 39. London: Athlone Press.

Reynolds, J. R. (1855) The Diagnosis of Diseases of the Brain, Spinal Cord, Nerves and their Appendages. London: Churchill.
Rush, B. (1812) Medical Inquiries and Observations upon the Diseases of the Mind. Philadelphia, PA: Kimber \& Richardson.

World Health Organization (1992) Internation Statistical Classification of Diseases and Related Health Problems. Geneva: WHO. 